\title{
Some Properties of Tests for Parameters That Can Be Arbitrarily
}

\section{Close to Being Unidentified}

\author{
by \\ G. Forchini ${ }^{12}$ \\ Monash University
}

March 24, 2009

\begin{abstract}
Confidence intervals for parameters that can be arbitrarily close to being unidentified are unbounded with positive probability (e.g. Dufour, Econometrica 65, pp. 1365-1387 and Pfanzagl, Journal of Statistical Planning and Inference, 75, pp. 9-20), and the asymptotic risks of their estimators are unbounded (Pötscher, Econometrica 70, pp. 1035-1065). We extend these "impossibility results" and show that all tests of size $\alpha$ concerning parameters that can be arbitrarily close to being unidentified have power that can be as small as $\alpha$ for any sample size even if the null and the alternative hypotheses are not adjacent. The results are proved for a very general framework that contains commonly used models.
\end{abstract}

\footnotetext{
${ }^{1}$ Address for correspondence: Giovanni Forchini, Department of Econometrics and Business Statistics, Monash University, Clayton, Victoria 3800, Australia.

E-mail: Giovanni.Forchini@BusEco.monash.edu.au

${ }^{2}$ I thank Grant Hillier, Patrick Marsh, Don Poskitt and Richard Smith for useful and encouraging comments and discussions. I thank the editor John Stufken and a referee for very useful feedback. This research was partially supported by Australian Research Council grant DP0771445.

Key Words: similar tests, consistent tests, uniformly consistent tests, identification, parameters arbitrarily close to being unidentified

AMS 2000 subject classifications: 62F03, 62G10
} 


\section{Introduction}

Models where the parameters can be arbitrarily close to being unidentified have recently attracted attention in both statistics (e.g. Gleser and Hwang (1987), Koschat (1987) and Pfanzagl (1998)) and econometrics (e.g. Staiger and Stock (1997), Dufour (1997), Andrews, Moreira and Stock (2006)). They are characterized by non-standard inferential problems. Gleser and Hwang (1987), Koschat (1987), Dufour (1997) and Pfanzagl (1998) have shown that every confidence set of level $\alpha$ must be unbounded with positive probability in these models. This suggests that the use of confidence intervals to measure the precision of an estimator may be problematic even when the sample size is very large (cf. Bahadur and Savage (1956), Singh (1963) and Pötscher (2002)). The results of LeCam and Schwartz (1960) and Pötscher (2002) suggest that no uniformly consistent estimator exists for parameters that can be arbitrarily close to being unidentified. When identification fails, standard estimators have non-standard asymptotic distributions. For structural equations models, this is shown by Phillips (1989), Choi and Phillips (1992), Staiger and Stock (1997), and Stock and Wright (2000).

Another challenging problem is the construction of (asymptotically) similar tests (i.e. tests with fixed size) for parameters that can be arbitrarily close to being unidentified. Dufour (1997) proves that tests based on Wald confidence sets cannot be similar, and that the sizes of such tests cannot be bounded from above in a nontrivial way. Kleibergen (2002) and Moreira (2003) show that similar tests for the structural parameters of linear structural equations do exist. The current interest in the construction of similar tests for these non-standard set-ups is evident from the long list of recent articles and working papers (e.g. Kleibergen (2005), Guggenberger and Smith (2005), Andrews and Marmer 
(2008), Andrews and Stock (2007), Andrews, Moreira and Stock (2006), Poskitt and Skeels (2005)).

In this paper we extend the impossibility results of Dufour (1997), Pfanzagl (1998) and Pötscher (2002) to tests of hypotheses, to help understand what optimal properties a test can have in situations where the parameters can be arbitrarily close to being unidentified. Our results are as follows:

(i) the power of a test of size $\alpha$ concerning a parameter that can be arbitrarily close to being unidentified can be as small as $1-\alpha$ for any sample size and for nonadjacent null and alternative hypotheses;

(ii) no test for which the asymptotic size is bounded by $0<\alpha<1$ can be uniformly consistent.

The source of the inferential problems about parameters that can be arbitrarily close to being unidentified is the discontinuity of the functional defining the parameter of interest at a point in the space of probability measures, as in Dufour (1997), Pfanzagl (1998) and Pötscher (2002). We characterise the problem by showing that the closures of the sets of probability measures under the null and alternative hypotheses have a nonempty intersection, even if the closures of the sets of the values that the parameter of interest can take on under the null and alternative hypotheses do have empty intersection. Our analysis is different from that of Romano (2004), who studies the case where the set of probability measures under the null hypothesis is dense in the set of probability measures under the alternative hypothesis. It is also different from the case occurring in time series where sets of probability measures under the null and alternative hypotheses are dense in a common set. Examples of the latter situation are tests of the spectral 
density at the origin (e.g. Blough (1992), Faust (1996) and Pötscher (2002)) or on long memory parameters (e.g. Pötscher (2002)). Usually in such cases one can impose restrictions on the relevant set of probability measures to eliminate the discontinuity of the functional of interest (e.g. Pötscher (2002)). For the case we consider, the discontinuity cannot be removed by restricting the set over which the functional of interest is defined, unless one assumes that the functional is identified and far from being unidentified.

We also investigate the power of an unbiased test for a parameter that can be arbitrarily close to being unidentified. We present an expression for the local power envelope, and show that the Fisher information is the key factor determining the largest power achievable by the test in a neighbourhood of the null hypothesis.

The remainder of the paper is structured as follows. Section 2 considers a very general set-up and some commonly used special cases (including the linear structural equation model), explains the notation, and derives and discusses some impossibility results. Section 3 bounds the local power of unbiased similar tests on a parameter that is close to being unidentified, and Section 4 concludes.

\section{Main results}

Let $\mathfrak{P}$ be a family of probability measures on a measurable space $(X, \mathcal{A})$. No assumption about the absolute continuity of the probability measures in $\mathfrak{P}$ is required (cf. Dufour (1997)). For any two probability measures $P$ and $Q$ in $\mathfrak{P}$, define the total variation distance between them as $d(P, Q)=\sup \{|P(A)-Q(A)|: A \in \mathcal{A}\}$, and let 
$\kappa: \mathfrak{P} \rightarrow \mathbb{R}$ be the functional defining the parameter of interest. Following Pfanzagl (1998), let $P_{*}$ be a probability measure, not necessarily in $\mathfrak{P}$, and let

$$
\begin{aligned}
& \mathfrak{P}_{\varepsilon}\left(P_{*}\right)=\left\{P \in \mathfrak{P}: d\left(P, P_{*}\right)<\varepsilon\right\} \\
& K_{\varepsilon}\left(P_{*}\right)=\kappa\left(\mathfrak{P}_{\varepsilon}\left(P_{*}\right)\right)
\end{aligned}
$$

and

$$
K\left(P_{*}\right)=\bigcap_{\varepsilon>0} K_{\varepsilon}\left(P_{*}\right) .
$$

We will define the parameter $\kappa$ as arbitrarily close to being unidentified using the notation above.

Definition. The parameter $\kappa$ is arbitrarily close to being unidentified if there exists a probability measure $P_{*}$ such that $K\left(P_{*}\right)=\kappa(\mathfrak{P})$, where this is a proper interval.

This implies that the functional $\kappa(P)$ can take on a range of values for probability measures $P$ arbitrarily close to $P_{*}$, and it is thus discontinuous at $P_{*}$. Examples of statistical models where a parameter of interest is arbitrarily close to being unidentified are given below.

Example 1. (See Dufour (1997) and Staiger and Stock (1997).) Consider the simple linear structural equation

$$
y_{t}=x_{t} \kappa+u_{t}
$$

with corresponding reduced form

$$
\left(y_{t}, x_{t}\right)=z_{t}^{\prime}(\pi, \gamma)+\left(w_{t}, v_{t}\right),
$$

$t=1,2, \ldots ., T$, where $y_{t}$ and $x_{t}$ are endogenous variables, $z_{t}$ is a $k$-dimensional vector of exogenous variables, $\kappa$ is a univariate structural parameter, and $\gamma$ and $\pi$ are $k$ - 
variate reduced form parameters. Moreover, $u_{t}, w_{t}$ and $v_{t}$ are random errors. Compatibility of (1) and (2) implies that $\pi=\gamma \kappa$ and $u_{t}=w_{t}-v_{t} \kappa$. Thus, the parameter $\kappa$ is uniquely defined (i.e. identified) in terms of the reduced form parameters when $\gamma \neq 0$. If $\gamma=0$ and equations (1) and (2) are satisfied, then $\kappa$ can take on any value, and is thus unidentified. This case is ruled out by assumption, but $\gamma$ is allowed to be arbitrarily close to zero: as $\gamma$ tends to zero. For example, in the case where $k=1$, $\kappa=\pi / \gamma$ and it can take on any value depending on the assumed behaviour of $\pi$.

Given that the structural and reduced form errors are linearly related, we can make assumptions on the properties of $\left(w_{t}, v_{t}\right), t=1,2, \ldots ., T$ only. For the sake of simplicity we assume that $\left(w_{t}, v_{t}\right), t=1,2, \ldots ., T$, are independent normal random variables with zero mean and $(2 \times 2)$ covariance matrix $\Sigma$. Let the joint probability distribution of the matrix variate $\left\{\left(y_{t}, x_{t}\right)\right\}_{t=1, \ldots, T}$ be indexed by $\pi, \gamma, \Sigma$ as $P_{\pi, \gamma, \Sigma}$. This can be written in terms of the parameters as $P_{\gamma \kappa, \gamma, \Sigma}=\tilde{P}_{\kappa, \gamma, \Sigma}$, so that

$$
\mathfrak{P}=\left\{\tilde{P}_{\kappa, \gamma, \Sigma}: \kappa \in \mathbb{R}, \gamma \neq 0, \Sigma \text { symmetric and positive definite }\right\}
$$

The probability $P_{*}$ can be any of the probabilities $\tilde{P}_{\kappa, 0, \Sigma}$. Notice that $P_{*} \notin \mathfrak{P}$, and that the functional $\kappa$ is arbitrarily close to being unidentified even for $\gamma \neq 0$.

Example 1 can be generalized to the case where $\left(w_{t}, v_{t}\right), t=1,2, \ldots ., T$, have arbitrary distributions that do not depend on the structural or reduced form parameters, and to the case where there are several structural parameters.

Example 2. Monte Carlo tests in their simplest form (e.g. Dufour (2006)) can be used to test the null hypothesis that $\kappa=\kappa_{0}$ and $P_{\kappa_{0}}$ is completely specified. Suppose that under the alternative, $\kappa=\kappa_{1} \neq \kappa_{0}$ and the distribution is $Q_{\kappa, \eta}=(1-\eta) P_{\kappa}+\eta P$, where $P$ is an 
arbitrary distribution in a set $\mathfrak{P}_{\mathcal{o}}$ containing a sequence converging to $P_{\kappa_{0}}$. In this case $P_{*}=Q_{\kappa, 1}$ for any $\kappa$. Once again, the case where $\eta=1$ is usually ruled out by assumption, but $\eta$ can be arbitrarily close to one.

Example 3. (See Pfanzagl (1998), Bahadur and Savage (1956).) In section 4, Pfanzagl (1998) considers a family $\mathfrak{P}$ of probability measures on the Borel sets with positive and continuous Lebesgue density. Pfanzagl (1998) assumes that the parameter of interest is $\kappa(P)=\int x P(d x)$, which is defined on the subfamily of $\mathfrak{P}$ for which $\int|x| P(d x)<\infty$. This family is closed under convex combinations (i.e. $P_{\alpha}=(1-\alpha) P_{*}+\alpha P \in \mathfrak{P}$ for $\alpha \in[0,1]$ if $\left.P_{*}, P \in \mathfrak{P}\right)$. For the functional considered, this implies that $\kappa\left(P_{\alpha}\right)=(1-\alpha) \kappa\left(P_{*}\right)+\alpha \kappa(P)$. Pfanzagl (1998) shows that $K\left(P_{*}\right)=\mathbb{R}=\kappa(\mathfrak{P})$.

We wish to test the null hypothesis $H_{0}: \kappa(P) \in \mathcal{H}_{0}$ against the alternative $H_{1}: \kappa(P) \in \mathcal{H}_{1}$, where $\mathcal{H}_{0}$ and $\mathcal{H}_{1}$ are two disjoint subsets of $\mathbb{R}$. We will make the following assumption:

Assumption 1. $\mathcal{H}_{0}$ and $\mathcal{H}_{1}$ are two disjoint subsets of $\kappa(\mathfrak{P})\left(\right.$ i.e. $\left.\mathcal{H}_{0} \cap \mathcal{H}_{1}=\varnothing\right)$ that are not adjacent (i.e. $\overline{\mathcal{H}}_{0} \cap \overline{\mathcal{H}}_{1}=\varnothing$, where $\overline{\mathcal{H}}_{i}$ denotes the closure of $\left.\mathcal{H}_{i}, i=1,2\right)$.

Our first result shows that if the parameter $\kappa$ can be arbitrarily close to being unidentified, then $\kappa^{-1}\left(\mathcal{H}_{0}\right)$ and $\kappa^{-1}\left(\mathcal{H}_{1}\right)$ can be arbitrarily close, even if the null and alternative hypotheses are not adjacent as required by Assumption 1. Precisely, 
Lemma 1. Suppose that $\kappa$ is arbitrarily close to being unidentified and let $\mathcal{H}_{0}$ and $\mathcal{H}_{1}$ be two arbitrary subsets of $\kappa(\mathfrak{P})$ that satisfy Assumption 1. Define the distance between $\kappa^{-1}\left(\mathcal{H}_{0}\right)$ and $\kappa^{-1}\left(\mathcal{H}_{1}\right)$ as

$$
D\left(\kappa^{-1}\left(\mathcal{H}_{0}\right), \kappa^{-1}\left(\mathcal{H}_{1}\right)\right)=\inf _{P \in \kappa^{-1}\left(\mathcal{H}_{0}\right), Q \in \kappa^{-1}\left(\mathcal{H}_{1}\right)} d(P, Q)
$$

Then, $D\left(\kappa^{-1}\left(\mathcal{H}_{0}\right), \kappa^{-1}\left(\mathcal{H}_{1}\right)\right)=0$.

Proof. Since $\kappa$ is arbitrarily close to being unidentified there is a probability measure $P_{*}$, which may or may not be in $\mathfrak{P}$, such that $K\left(P_{*}\right)=\kappa(\mathfrak{P})$. Let $\mathcal{H}$ be an arbitrary subset of $\kappa(\mathfrak{P})$. Since $\kappa(\mathfrak{P})=K\left(P_{*}\right)=\bigcap_{\varepsilon>0} K_{\varepsilon}\left(P_{*}\right)$, we must have that $\mathcal{H} \subset K_{\varepsilon}\left(P_{*}\right)$ for every $\varepsilon>0$. Therefore, for every $\mathcal{H} \subset \kappa(\mathfrak{P})$ and every $\varepsilon>0$ there is a probability measure $P \in \kappa^{-1}(\mathcal{H})$ such that $d\left(P, P_{*}\right)<\varepsilon$. Taking $\mathcal{H}_{0}$ and $\mathcal{H}_{1}$ as two subsets of $\kappa(\mathfrak{P})$ and using the triangle inequality, we have that

$$
\begin{aligned}
D\left(\kappa^{-1}\left(\mathcal{H}_{0}\right), \kappa^{-1}\left(\mathcal{H}_{1}\right)\right) & \leq \inf _{P \in \kappa^{-1}\left(\mathcal{H}_{0}\right), Q \in \kappa^{-1}\left(\mathcal{H}_{4}\right)}\left(d\left(P, P_{*}\right)+d\left(P_{*}, Q\right)\right) \\
& \leq \varepsilon+\varepsilon \\
& =2 \varepsilon .
\end{aligned}
$$

The result follows from the fact that $\varepsilon$ is an arbitrary positive number.

Lemma 1 is a simple consequence of the definition of a parameter close to being unidentified. It isolates the problems with constructing tests in such a set-up: even though the null and alternative hypotheses are not adjacent, their inverse images are adjacent. 
Because of this, one would expect all tests to have difficulty in distinguishing between the null and alternative hypotheses. We will now show that this is what happens.

Let $\hat{\varphi}: X \rightarrow[0,1]$ be a test of the null hypothesis against the alternative (i.e. $\hat{\varphi}(x)$ is a measurable function from $X$ to $[0,1])$. The main result of this paper can now be stated.

Theorem 1. Suppose that the parameter $\kappa$ is arbitrarily close to being unidentified and let $\hat{\varphi}(x)$ be a test of the null hypothesis $H_{0}: \kappa(P) \in \mathcal{H}_{0}$ against the alternative $H_{1}: \kappa(P) \in \mathcal{H}_{1}$. Then,

$$
\inf _{P \in \kappa^{-1}\left(\mathcal{H}_{1}\right)} E_{P}[\hat{\varphi}(x)] \leq \sup _{P \in \kappa^{-1}\left(\mathcal{H}_{0}\right)} E_{P}[\hat{\varphi}(x)]
$$

If $\hat{\varphi}(x)$ has size $\alpha$ (i.e. $\sup _{P \in \mathcal{K}^{-1}\left(\mathcal{H}_{0}\right)} E_{P}[\hat{\varphi}(x)] \leq \alpha$ ), then

$$
\inf _{P \in \kappa^{-1}\left(\mathcal{H}_{1}\right)} E_{P}[\hat{\varphi}(x)] \leq \alpha .
$$

Proof. Since $\kappa$ is arbitrarily close to being unidentified there is a probability measure $P_{*}$, which may or may not be in $\mathfrak{P}$, such that $K\left(P_{*}\right)=\kappa(\mathfrak{P})$. As in the proof of Lemma 1, we can find $P_{i} \in \kappa^{-1}\left(\mathcal{H}_{i}\right), i=0,1$, s such that $d\left(P_{i}, P_{*}\right)<\varepsilon / 2$ for $i=0,1$. Since $0 \leq \hat{\varphi}(x) \leq 1$, we have that $\left|E_{P_{1}} \hat{\varphi}(x)-E_{P_{0}} \hat{\varphi}(x)\right| \leq d\left(P_{1}, P_{0}\right)$. That is,

$$
E_{P_{1}} \hat{\varphi}(x) \leq E_{P_{0}} \hat{\varphi}(x)+d\left(P_{1}, P_{0}\right) .
$$

The left hand side is at least as large as $\inf _{P \in \kappa^{-1}\left(\mathcal{H}_{1}\right)} E_{P}[\hat{\varphi}(x)]$, while the expectation on the right hand side is at most $\sup _{P \in \kappa^{-1}\left(\mathcal{H}_{0}\right)} E_{P}[\hat{\varphi}(x)]$. Moreover 


$$
d\left(P_{1}, P_{0}\right) \leq d\left(P_{0}, P_{*}\right)+d\left(P_{1}, P_{*}\right)<\varepsilon .
$$

Thus

$$
\inf _{P \in \kappa^{-1}\left(\mathcal{H}_{1}\right)} E_{P}[\hat{\varphi}(x)] \leq \sup _{P \in \kappa^{-1}\left(\mathcal{H}_{0}\right)} E_{P}[\hat{\varphi}(x)]+\varepsilon .
$$

The inequality in (3) follows from the fact that $\varepsilon$ is arbitrary. If $\hat{\varphi}(x)$ has size $\alpha$, then $\sup _{P \in \kappa^{-1}\left(\mathcal{H}_{0}\right)} E_{P}[\hat{\varphi}(x)] \leq \alpha$ and, (4) follows. This completes the proof.

Theorem 1 covers the case where the null and alternative hypotheses are adjacent $\left(\overline{\mathcal{H}}_{0} \cap \overline{\mathcal{H}}_{1} \neq \varnothing\right)$ as well as the case where the null and alternative hypotheses are not adjacent $\left(\overline{\mathcal{H}}_{0} \cap \overline{\mathcal{H}}_{1}=\varnothing\right)$. In the first case, the result is not new and it follows from the continuity of the power as a function of the underlying probability measures. It is the second case, in which Assumption 1 holds, that makes the result interesting and new.

Theorem 1 complements the impossibility results of Dufour (1997), Pfanzagl (1998) and Pötscher (2002) and shows that, even if the null and the alternative hypotheses are not adjacent, all similar tests have potentially low power. The reason for this loss of power hinges on the fact that the inverse images of $\mathcal{H}_{0}$ and $\mathcal{H}_{1}$ are adjacent for all $\mathcal{H}_{0}$ and $\mathcal{H}_{1}$, as illustrated in Lemma 1 . This results in tests having the same properties as tests in the standard set-up where the null and the alternative hypotheses are adjacent.

It follows from equation (4) that the probability of type II errors of any test satisfies

$$
\sup _{P \in \kappa^{-1}\left(\mathcal{H}_{\mathcal{H}}\right)}\left(1-E_{P}[\hat{\varphi}(x)]\right)=1-\inf _{P \in \kappa^{-1}\left(\mathcal{H}_{1}\right)} E_{P}[\hat{\varphi}(x)] \geq 1-\alpha .
$$


Therefore, all similar tests of size $\alpha$ of $H_{0}: \kappa(P) \in \mathcal{H}_{0}$ against $H_{1}: \kappa(P) \in \mathcal{H}_{1}$ can have probabilities of type II errors larger than $1-\alpha$.

Notice that the problems persist in an asymptotic framework. Let $(X, \mathcal{A})=\left(X_{1} \times X_{2} \times \ldots \times X_{n}, \mathcal{A}_{1} \times \mathcal{A}_{2} \times \ldots \times \mathcal{A}_{n}\right)$, where $n$ is an index that may denote the sample size, and we denote by $\hat{\varphi}_{n}(x)$ for $x \in X_{1} \times X_{2} \times \ldots \times X_{n}$ a test of the null hypothesis $H_{0}: \kappa(P) \in \mathcal{H}_{0}$ against the alternative hypothesis $H_{1}: \kappa(P) \in \mathcal{H}_{1}$. Suppose that $\mathcal{H}_{0}$ and $\mathcal{H}_{1}$ satisfy Assumption 1 and the parameter $\kappa$ is arbitrarily close to being unidentified. If $\hat{\varphi}_{n}(x)$ has size $\alpha$ as $n \rightarrow \infty$ (i.e. $\limsup _{n \rightarrow \infty} \sup _{P \in \kappa^{-1}\left(\mathcal{H}_{0}\right)} E_{P}\left[\hat{\varphi}_{n}(x)\right]=\alpha$ ), then, by taking the limit as $n$ tends to infinity in (3), we have $\limsup _{n \rightarrow \infty} \inf _{P \in \kappa^{-1}\left(\mathcal{H}_{1}\right)} E_{P}\left[\hat{\varphi}_{n}(x)\right] \leq \alpha$. Clearly, $E_{P}\left[\hat{\varphi}_{n}(x)\right]$ does not converge to one uniformly over $\kappa^{-1}\left(\mathcal{H}_{1}\right)$ indicating that no test of $H_{0}: \kappa(P) \in \mathcal{H}_{0}$ against $H_{1}: \kappa(P) \in \mathcal{H}_{1}$ asymptotically having size $\alpha$ is uniformly consistent.

Our Theorem 1 is similar to Theorem 1 of Romano (2004), as is the method of proof. Romano (2004) shows that if the set $\kappa^{-1}\left(\mathcal{H}_{0}\right)$ is dense in $\kappa^{-1}\left(\mathcal{H}_{1}\right)$, then the power of any similar test of size $\alpha$ cannot exceed $\alpha$. We only require that $\kappa^{-1}\left(\mathcal{H}_{0}\right)$ and $\kappa^{-1}\left(\mathcal{H}_{1}\right)$ contain two sequences converging to the same limit, which is certainly the case when the functional $\kappa$ is close to being unidentified. 


\section{The power of tests on $\kappa$ in the neighbourhood of $P_{*}$}

We now investigate the dependence of the power of unbiased tests of $\kappa$ on the parameters affecting identification. The assumptions here are stronger than those in Section 2.

Consider a family of probability measures $\mathfrak{P}=\left\{P_{\kappa, \phi}: \kappa \in \mathbb{R}, \phi \in \Phi\right\}$, where $\Phi$ is an arbitrary metric space. It can be either a family of fully parametric models if $\Phi$ is an Euclidean space, or a family of semi-parametric models. Define the functional of interest as $\kappa\left(P_{\kappa, \phi}\right)=\kappa$, and suppose that the parameter $\kappa$ is identified (i.e. $P_{\kappa_{1}, \phi_{1}}=P_{\kappa_{2}, \phi_{2}}$ implies $\kappa_{1}=\kappa_{2}$ ). Moreover, assume that there exists $\phi_{*} \in \Phi$ and a probability measure $P_{*}$ on $(X, \mathcal{A})$, not necessarily in $\mathfrak{P}$, such that $\lim _{\phi \rightarrow \phi_{*}} d\left(P_{\kappa, \phi}, P_{*}\right)=0$ for all $\kappa \in \mathbb{R}$. Thus, $\kappa$ is arbitrarily close to being unidentified. We also make the following assumption which allows us to locally express the Hellinger distance as a quadratic form (Blyth (1994)):

Assumption 2. $P_{\kappa, \phi}$ is absolutely continuous with respect to a dominating measure $\mu$ with density $p(x ; \kappa, \phi)$; moreover

(i) The set $\{x: p(x ; \kappa, \phi)>0\}$ does not depend on $(\kappa, \phi)$;

(ii) Partial differentiation with respect to $(\kappa, \phi)$ and integration with respect to $x$ of the functions $h(x,(\kappa, \phi))$ are interchangeable whenever required; and (iii) The Fisher information matrix exists and is finite. 
The Fisher information about $\kappa$ for a fixed $\phi$ is $I_{\kappa}(\phi)=E_{P_{\kappa, \phi}}\left[\dot{\ell}_{\kappa,[\phi]}^{2}\right]$, where $\dot{\ell}_{\kappa,[\phi]}$ denotes the partial derivative of $\ell_{\kappa, \phi}=\ln \left(P_{\kappa, \phi}\right)$ with respect to $\kappa$. Notice that at $\phi_{*}, \ell_{\kappa, \phi_{*}}$ does not depend on $\kappa$, so that both $\dot{\ell}_{\kappa,\left[\phi_{*}\right]}^{2}=0$ and $I_{\kappa}\left(\phi_{*}\right)=0$.

Theorem 2. Suppose that Assumption 2 holds and that $E_{P_{\kappa, \phi}} \hat{\varphi}(x)$ is smooth in the sense that it has derivatives of order $k \geq 2$. Then, the power of every unbiased test $\hat{\varphi}(x)$ of size $\alpha$ (i.e. such that $\left.\sup _{P \in \kappa^{-1}\left(\mathcal{H}_{0}\right)} E_{P}[\hat{\varphi}(x)] \leq \alpha \leq \sup _{P \in \kappa^{-1}\left(\mathcal{H}_{1}\right)} E_{P}[\hat{\varphi}(x)]\right)$ for $H_{0}: \kappa=\kappa_{0}$ against $H_{1}: \kappa=\kappa_{0}+\delta(\delta \neq 0)$ satisfies

$$
\left.\frac{d E_{P_{\kappa, \phi}} \hat{\varphi}(x)}{d \kappa}\right|_{\kappa=\kappa_{0}}=0
$$

and

$$
0<\left.\frac{d^{2} E_{P_{\kappa, \phi}} \hat{\varphi}(x)}{d \kappa^{2}}\right|_{\kappa=\kappa_{0}} \leq I_{\kappa_{0}}(\phi) .
$$

Thus, the power of every unbiased similar test $\hat{\varphi}(x)$ for $H_{0}: \kappa=\kappa_{0}$ against $H_{1}: \kappa=\kappa_{0}+\delta(\delta \neq 0)$ satisfies

$$
E_{P_{\kappa_{0}+\delta, \phi}} \hat{\varphi}(x) \leq \alpha+(1 / 2) I_{\kappa_{0}}(\phi)(\tau(\delta))^{2} \delta^{2}
$$

in a neighbourhood of $\delta=0$, where $\tau: \mathbb{R} \rightarrow \mathbb{R}$ is the function

$$
\tau(\delta)=\sqrt{\frac{\left.\int_{0}^{1} \int_{0}^{t_{1}} \frac{d^{2} E_{P_{\kappa, \phi}} \hat{\varphi}(x)}{d \kappa^{2}}\right|_{\kappa_{0}+t \delta} d t d t_{1}}{\left.\frac{1}{2} \frac{d^{2} E_{P_{\kappa, \phi}} \hat{\varphi}(x)}{d \kappa^{2}}\right|_{\kappa_{0}}}} .
$$


Proof. Let $f(\delta)=E_{P_{\kappa_{0}+\delta, \phi}} \hat{\varphi}(x)-E_{P_{\kappa_{0}, \phi}} \hat{\varphi}(x)$ for fixed $\kappa_{0}$ and $\phi$. Such function has the following properties:

1. $f(0)=0$.

2. $f(\delta)>0$ for $\delta \neq 0$ since the tests considered are in unbiased.

3. For any $\delta$, the first derivative is

$$
\frac{d f(\delta)}{d \delta}=\lim _{h \rightarrow 0} \frac{f(\delta+h)-f(\delta)}{h}=\lim _{h \rightarrow 0} \frac{E_{P_{\kappa_{0}+\delta+t h, \phi}} \hat{\varphi}(x)-E_{P_{\kappa_{0}+\delta, \phi}} \hat{\varphi}(x)}{h}=\frac{d E_{P_{\kappa_{0}+\delta, \phi}} \hat{\varphi}(x)}{d \delta} .
$$

4. At $\delta=0$ the first derivative is zero. It is well known that

$$
f(\delta)=E_{P_{\kappa_{0}+\delta, \phi}} \hat{\varphi}(x)-E_{P_{\kappa_{0}, \phi}} \hat{\varphi}(x) \leq(1 / 2) d\left(P_{\kappa_{0}, \phi}, P_{\kappa_{0}+\delta, \phi}\right) \leq H\left(P_{\kappa_{0}, \phi}, P_{\kappa_{0}+\delta, \phi}\right),
$$

where $H$ denotes the Hellinger distance. Assumption 2 guarantees that the Hellinger distance satisfies $H\left(P_{\kappa_{0}, \phi}, P_{\kappa_{0}+\delta, \phi}\right)=(1 / 2) I_{\kappa_{0}}(\phi) \delta^{2}+O\left(\delta^{3}\right)$ in a neighbourhood of $\delta=0$ (e.g. Blyth (1994)). Then, for $\delta$ tending to zero from the right

$$
0<\lim _{\delta \rightarrow 0_{+}} \frac{f(\delta)-f(0)}{\delta}=\lim _{\delta \rightarrow 0_{+}} \frac{f(\delta)}{\delta} \leq \lim _{\delta \rightarrow 0_{+}}(1 / 2) I_{\kappa_{0}}(\phi) \delta=0,
$$

and for $\delta$ tending to zero from the left

$$
0=\lim _{\delta \rightarrow 0_{-}}(1 / 2) I_{\kappa_{0}}(\phi) \delta \leq \lim _{\delta \rightarrow 0_{-}} \frac{f(\delta)-f(0)}{\delta}=\lim _{\delta \rightarrow 0_{-}} \frac{f(\delta)}{\delta}<0,
$$

so that $\left.\frac{d f(\delta)}{d \delta}\right|_{\delta=0}=\left.\frac{d E_{P_{\kappa_{0}+\delta, \phi}} \hat{\varphi}(x)}{d \delta}\right|_{\delta=0}=0$. 
5. Proceeding as in 3 one can easily check that $\frac{d^{2} f(\delta)}{d \delta^{2}}=\frac{d^{2} E_{P_{x_{0}+\delta, \phi}} \hat{\varphi}(x)}{d \delta^{2}}$.

6. At $\delta=0$ the second derivative is bounded by the Fisher information. Proceeding as in 4, we find that $0<\lim _{\delta \rightarrow 0} \frac{E_{P_{\kappa_{0}, \phi}} \hat{\varphi}(x)-E_{P_{\kappa_{0}+\delta, \phi}} \hat{\varphi}(x)}{\delta^{2}} \leq(1 / 2) I_{\kappa_{0}}(\phi)$. Applying L'Hospital's rule we have

$$
\begin{aligned}
\lim _{\delta \rightarrow 0} \frac{E_{P_{\kappa_{0}+\delta, \phi}} \hat{\varphi}(x)-E_{P_{\kappa_{0}, \phi}} \hat{\varphi}(x)}{\delta^{2}} & =\lim _{\delta \rightarrow 0} \frac{\frac{d\left(E_{P_{\kappa_{0}+\delta, \phi}} \hat{\varphi}(x)-E_{P_{\kappa_{0}, \phi}} \hat{\varphi}(x)\right)}{\frac{d \delta}{d \delta}}}{} \\
& =\lim _{\delta \rightarrow 0} \frac{\frac{d E_{P_{\kappa_{0}+\delta, \phi}} \hat{\varphi}(x)}{d \delta}}{2 \delta} \\
& =\frac{1}{2} \lim _{\delta \rightarrow 0} \frac{\left.\frac{d E_{P_{\kappa, \phi}} \hat{\varphi}(x)}{d \kappa}\right|_{\kappa=\kappa_{0}+\delta}-0}{\delta}=\left.\frac{1}{2} \frac{d^{2} E_{P_{\kappa, \phi}} \hat{\varphi}(x)}{d \kappa^{2}}\right|_{\kappa=\kappa_{0}},
\end{aligned}
$$

So that $0<\left.\frac{d^{2} E_{P_{\kappa_{0}+\delta, \phi}} \hat{\varphi}(x)}{d \delta^{2}}\right|_{\delta=0}=\left.\frac{d^{2} f(\delta)}{d \delta^{2}}\right|_{\delta=0} \leq(1 / 2) I_{\kappa_{0}}(\phi)$.

Now we establish (8) by specializing some results in Chapter 1 of the book by Castrigiano and Hayes (1993). Since $\quad f(0)=\left.\frac{d f(\delta)}{d \delta}\right|_{\delta=0}=0 \quad$ and $0<\left.\frac{d^{2} f(\delta)}{d \delta^{2}}\right|_{\delta=0} \leq I_{\kappa_{0}}(\phi)$, such a function can be written as $f(\delta)=g(\delta) \delta^{2}$, where $g(\delta)=\left.\int_{0}^{1} \int_{0}^{t_{1}} \frac{d^{2} f(q)}{d q^{2}}\right|_{q=t \delta} d t d t_{1}$ is a smooth function (cf. Theorem 25 of Castrigiano and 
Hayes (1993)). One can easily check that $\left.\frac{1}{2} \frac{d^{2} f(\delta)}{d \delta^{2}}\right|_{\delta=0}=g(0)$. Since $g(\delta)$ is smooth around $\delta=0$, there is an open interval containing zero, such that $g(\delta)=g(0)[\tau(\delta)]^{2}$, where

$$
\tau(\delta)=\sqrt{\frac{\left.\int_{0}^{1} \int_{0}^{t_{1}} \frac{d^{2} f(q)}{d q^{2}}\right|_{q=t \delta} d t d t_{1}}{\left.\int_{0}^{t_{1}} \frac{d^{2} f(q)}{d q^{2}}\right|_{q=0} d t d t_{1}}},
$$

which simplifies to (9), is a smooth function such that $\tau(0)=1$ (cf. Theorem 36 of Castrigiano and Hayes (1993)). Thus, in a neighbourhood of $\delta=0$ we can write $f(\delta)=g(0)[\tau(\delta)]^{2} \delta$ and $f(\delta) \leq \frac{1}{2} I_{\kappa_{0}}(\phi)[\tau(\delta)]^{2} \delta^{2}$. Equation (8) follows by noticing that, since the tests considered are similar, $\sup _{P \in \kappa^{-1}\left(\mathcal{H}_{0}\right)} E_{P}[\hat{\varphi}(x)]=\alpha$.

If $I_{\kappa}(\phi)$ is a continuous function of $\phi$ for $\phi \neq \phi_{*}$, then $I_{\kappa}(\phi) \rightarrow I_{\kappa}\left(\phi_{*}\right)=0$ as $\phi \rightarrow \phi_{*}$ for all $\kappa$. Clearly, if $\kappa$ is unidentified the power of every similar test is bounded from above by $\alpha$. Therefore, if $I_{\kappa_{0}}(\phi)$ is large we may find tests of $H_{0}: \kappa=\kappa_{0}$ versus $H_{1}: \kappa=\kappa_{0}+\delta(\delta \neq 0)$ that are locally unbiased, but if $I_{\kappa_{0}}(\phi)$ is small all similar tests will have very low local power.

Theorem 2 is a version of Theorem 1 of Marsh (2007) that allows for nuisance parameters and a more general class of probability measures. Notice also that the local upper bound for the power given by Marsh (2007) would be, in our notation, 
$\alpha+I_{\kappa_{0}+\delta_{*}}(\phi) \delta^{2}$, where $\delta_{*}$ is a number between $\kappa_{0}$ and $\delta$, while, in our Theorem 2, the information is evaluated at the null hypothesis. Asymptotic versions of this result in the case where there are no nuisance parameters and $P_{\kappa}$ is normal are well known (e.g. Section 15.3 of Van der Vaart (1998)). If $I_{\kappa_{0}}(\phi)$ is zero, the power envelope is described locally by a more complicated function.

Our "trust" in tests on parameters that can be arbitrarily close to being unidentified must rely on information about $I_{\kappa_{0}}(\phi)$, which can be interpreted as half the curvature of the power function at $\kappa_{0}$. Given some regularity conditions, $I_{\kappa_{0}}(\phi)$ can be consistently estimated for each fixed $\phi \neq \phi_{0}$. We have argued (see Forchini and Hillier (2003)) that inference should be conditional on a measure of distance from the critical set where the parameter of interest is unidentified. The quantity $I_{\kappa_{0}}(\phi)$, or an estimate thereof, is certainly a measure of identification (and one may argue for conditioning on it).

\section{Conclusions}

In this paper, we have shown that tests on parameters arbitrarily close to being unidentified cannot satisfy some of the properties that are usually expected from tests in a standard set-up when the null and alternative hypotheses are not adjacent. These results complement those of Dufour (1997), Pfanzagl (1998) and Pötscher (2002) on confidence intervals and point estimators.

We have shown that every test of parameters arbitrarily close to being unidentified that has size bounded from above by a known constant has potentially low power and a large probability of type II errors. Since models with parameters arbitrarily close to being 
unidentified seem to be very frequent in practical applications, our main result, Theorem 1, adds to the existing evidence that standard optimality criteria for tests may be inadequate tools to deal with these situations. Theorem 2 provides a local upper bound for the power of tests of the null hypothesis $H_{0}: \kappa=\kappa_{0}$ in terms of the Fisher information about $\kappa$ for a fixed nuisance parameter.

\section{References}

Andrews, D. and V. Marmer (2008). "Exactly Distribution-Free Inference in Instrumental Variables Regression with Possibly Weak Instruments." Journal of Econometrics, forthcoming.

Andrews, D. and J. H. Stock (2007). Inference with Weak Instruments. Advances in Economics and Econometrics, Theory and Applications: Ninth World Congress of the Econometric Society, Cambridge University Press.

Andrews, D. W. K., M. J. Moreira and J. H. Stock (2006). "Optimal Two-sided Invariant Similar Tests for Instrumental Variables Regression." Econometrica 74: 715-752.

Bahadur, R. R. and L. J. Savage (1956). "The Nonexistence of Certain Statistical Procedures in Nonparametric Problems." Annals of Mathematical Statistics 27: 1115-1122.

Blough, S. R. (1992). "The Relationship Between Power and Level for Generic Unit Root Tests in Finite Samples." Jounal of Applied Econometrics 7: 295-308.

Blyth, S. (1994). "Local Divergence and Association." Biometrika 81: 579-584.

Castrigiano, D. P. L. and S. A. Hayes (1993). Catastrophe Theory. New York, AddisonWesley Publishing Company.

Choi, I. and P. C. B. Phillips (1992). "Asymptotic and Finite Sample Distribution Theory for IV Estimators and Tests in Partially Identified Structural Equations." Journal of Econometrics 51: 113-150.

Dufour, J.-M. (1997). "Some Impossibility Theorems in Econometrics with Applications to Instrumental Variables and Dynamic Models." Econometrica 65: 1365-1388. 
Dufour, J.-M. (2006). "Monte Carlo Tests with Nuisance Parameters: A General Approach to Finite-Sample Inference and Nonstandard Asymptotics." Journal of Econometrics 133(2): 443-477.

Faust, J. (1996). "Near Observational Equivalence and Theoretical Size Problems with Unit Root Tests." Econometric Theory 12: 724-731.

Forchini, G. and G. Hillier (2003). "Conditional Inference for Possibly Unidentified Structural Equations." Econometric Theory 19: 707-743.

Gleser, L. J. and J. T. Hwang (1987). "The Nonexistence of 100(1- $\alpha$ )." The Annals of Statistics 15: 1351-1362.

Guggenberger, P. and R. J. Smith (2005). "Generalized Empirical Likelihood Estimators and Tests under Partial, Weak and Strong Identification." Econometric Theory 21: 667-709.

Kleibergen, F. (2002). "Pivotal Statistics for Testing Structural Parameters in Instrumental Variables Regression." Econometrica 70: 1781-1803.

Kleibergen, F. (2005). "Testing Parameters in GMM Without Assuming that They Are Identified." Econometrica 73: 1103-1123.

Koschat, M. A. (1987). "A Characterization of the Fieller Solution." The Annals of Statistics 15: 462-468.

LeCam, L. and L. Schwartz (1960). "A Necessary and Sufficient Condition for the Existence of Consistent Estimates." The Annals of Mathematical Statistics 19: $140-150$.

Marsh, P. (2007). "The Available Information for Invariant Tests for a Unit Root." Econometric Theory 23(4): 686-685.

Moreira, M. J. (2003). "A Conditional Likelihood Ratio Test for Structural Models." Econometrica 71: 1027-1048.

Pfanzagl, J. (1998). "The Nonexistence of Confidence Sets for Discontinuous Functionals." Journal of Statistical Planning and Inference 75: 9-20.

Phillips, P. C. B. (1989). "Partially Identified Econometric Models." Econometric Theory 5: $181-240$. 
Poskitt, D. and C. Skeels (2005). "Small Concentration Asymptotics and Instrumental Variables Inference." Research Paper No. 948, Department of Economics, University of Melbourne.

Pötscher, B. M. (2002). "Lower Risk Bounds and Properties of Confidence Sets for IllPosed Estimation Problems with Applications to Spectral Density and Persistence Estimation, Unit Roots, and Estimation of Long Memory Parameters." Econometrica 70: 1035-1065.

Romano, J. P. (2004). "On Non-Parametric Testing, the Uniform Behaviour of the t-test, and Related Problems." The Scandinavian Journal of Statistics 65: 557-586.

Singh, R. (1963). "Existence of Bounded Length Confidence Intervals." Annals of Mathematical Statistics 34: 1474-1485.

Staiger, D. and J. H. Stock (1997). "Instrumental Variables Regression with Weak Instruments." Econometrica 65: 557-586.

Stock, J. H. and J. H. Wright (2000). "GMM with Weak Identification." Econometrica 68: 1055-1096.

Van der Vaart, A. W. (1998). Asymptotic Statistics. Cambridge, Cambridge University Press. 